\title{
Reevaluating the Roles of Eddies in Multiple Barotropic Wind-Driven Gyres
}

\author{
BAYLOR FOX-KEMPER \\ Massachusetts Institute of Technology-Woods Hole Oceanographic Institution Joint Program in Oceanography and Ocean \\ Engineering, Woods Hole, Massachusetts
}

(Manuscript received 3 December 2003, in final form 13 December 2004)

\begin{abstract}
Multiple-gyre ocean models have a weaker mean subtropical circulation than single-gyre calculations with the same viscosity and subtropical forcing. Traditionally, this reduction in circulation is attributed to an intergyre eddy vorticity flux that cancels some of the wind input, part of which does not require a Lagrangian mass exchange (theory of dissipative meandering). Herein the intergyre eddy vorticity flux is shown to be a controlling factor in barotropic models at high Reynolds number only with exactly antisymmetric gyres and slip boundary conditions. Almost no intergyre flux occurs when no-slip boundary conditions are used, yet the subtropical gyre is still significantly weaker in multiple-gyre calculations. Sinuous modes of instability present only in multiple gyres are shown here to vastly increase the eddy vorticity transport efficiency. This increase in efficiency reduces the mean circulation necessary for equilibrium. With slip boundary conditions, the intergyre eddy transport is possibly much larger. However, with wind forcing relevant for the ocean-two unequal gyres - a mean flow flux of vorticity rather than an eddy flux between the regions of opposing wind forcing is increasingly important with increasing Reynolds number. A physical rationalization of the differing results is provided by diagnosis of the equilibrium vorticity budget and eddy transport efficiency. Calculations varying 1) boundary conditions, 2) sources and sinks of vorticity, 3) eddy transport efficiency, and 4) the degree of symmetry of the gyres are discussed.
\end{abstract}

\section{Introduction}

Since the introduction of frictional western boundary current models by Stommel (1948) and Munk (1950), less viscous models capable of producing strongly western-intensified flows have been sought. Reducing the friction while maintaining a realistic circulation is a difficult task. The ocean is a forced-dissipative system, so purely inertial theories (e.g., Fofonoff 1954; Charney 1955; Carrier and Robinson 1962) are unable to close the budgets of energy and vorticity and determine the circulation strength for a wind-driven ocean.

This paper focuses on the mechanisms by which the vorticity budget ${ }^{1}$ is closed in a number of related highly

\footnotetext{
${ }^{1}$ The energy budget is not as easily handled, as energy input is solution dependent (Scott and Straub 1998) and energy fluxes are difficult to define (Plumb 1982). In practice, much of the energy analysis is redundant with vorticity analysis (Fox-Kemper 2003).

Corresponding author address: Baylor Fox-Kemper, Department of Earth, Atmospheric and Planetary Sciences, Massachusetts Institute of Technology, Cambridge, MA 01239.

E-mail: baylor@alum.mit.edu
}

nonlinear barotropic models. A contrast is drawn between the vorticity dynamics of models with single-gyre wind forcing (vorticity input always negative) and $m u l$ tiple-gyre wind forcing (vorticity input of both signs). Different boundary conditions and frictional operators are also shown to have meaningful effects. Analysis of the vorticity budget proves a powerful tool in the oceanically relevant parameter range far from the onset of instability.

A fixed-depth barotropic model is used, which obeys the nondimensional vorticity equation, (1):

$$
\frac{\partial \zeta}{\partial t}+\nabla \cdot\left(\hat{\mathbf{x}} \psi+\delta_{I}^{2} \mathbf{u} \zeta-\delta_{M}^{3} \nabla \zeta+\delta_{S} \nabla \psi\right)=w_{E},
$$

where

$$
\zeta=\nabla^{2} \psi
$$

The variables $\psi$ [streamfunction: $\mathbf{u} \equiv(-\partial \psi / \partial y, \partial \psi / \partial x)$ ] and $\zeta$ (relative vorticity) are determined during integration. The calculations are differentiated by the values of the parameters: $\delta_{I}$ (Charney 1955: inertial boundary layer width), $\delta_{S}$ (Stommel 1948: frictional boundary layer width), and $\delta_{M}$ (Munk 1950: frictional boundary layer width). A unit vector in the zonal direction is $\hat{\mathbf{x}}$. 
All of these widths are relative to the zonal basin extent. The time mean of (1) yields

$$
\nabla \cdot\left(\hat{\mathbf{x}} \bar{\psi}+\delta_{I}^{2} \overline{\mathbf{u}} \bar{\zeta}+\delta_{I}^{2} \overline{\mathbf{u}^{\prime} \bar{\zeta}^{\prime}}-\delta_{M}^{3} \nabla \bar{\zeta}+\delta_{S} \nabla \bar{\psi}\right)=\bar{w}_{E} .
$$

Overbar denotes a mean, and primes denote perturbations about it. Following Haynes and McIntyre (1987), note that (1) and (3) are in flux form, and so vorticity is neither created nor destroyed between its input by the wind $\left(w_{E}\right)$ and its removal through the basin walls by either lateral or bottom friction. The fluxes in the square brackets will be called respectively $\beta$ flux, mean flux, eddy flux, lateral friction flux, and bottom friction flux.

Consider for a moment a time mean calculated over a time interval longer than the eddy time scales, but shorter than the total postequilibration time interval. If the vorticity fluxes are not in balance, as during spinup, then a circulation adjustment must occur since the vorticity contained within a mean streamline (where $\bar{\psi}=$ $\psi_{c}$ ) is directly related to the circulation about the streamline, $\Gamma$ :

$$
|\Gamma| \equiv\left|\int_{\bar{\psi} \geq \psi_{c}} \nabla^{2} \bar{\psi} d A\right|=\left|\oint_{\psi_{c}} \nabla \bar{\psi} \cdot \hat{\mathbf{n}} d s\right|=|\langle\bar{u}\rangle| L .
$$

If the vorticity contained increases, either the mean velocity magnitude $(|\langle\bar{u}\rangle|)$ increases or the length of the mean streamline $(L)$ increases. (The angle brackets denote the average value along the streamline.) Vorticity trapped between two streamlines has a similar effect on the difference in circulation of the two streamlines. In this sense, the transport and pooling of vorticity controls the circulation strength. For the remainder of the paper, the time mean will be considered to be taken over the second half of the total integration, thus after equilibration.

Many fluxes play a role in balancing the vorticity budget within each streamline and within the entire basin. In the basin interior, away from the boundary currents and recirculations, the Sverdrup (1947) balance often dominates (i.e., the wind input balances the $\beta$ flux divergence). However, the $\beta$ flux cannot transport net vorticity across a closed mean streamline, as integrating (3) indicates:

$$
\oint_{\psi_{c}}\left(\delta_{I}^{2} \overline{\mathbf{u}^{\prime} \zeta^{\prime}}+\delta_{S} \nabla \bar{\psi}-\delta_{M}^{3} \nabla \bar{\zeta}\right) \cdot \hat{\mathbf{n}} d s=\iint_{\bar{\psi} \geq \psi_{c}} \bar{w}_{E} d A .
$$

The $\beta$ flux can affect the vorticity distribution within a mean streamline, but it cannot reduce or increase the total. The same is true for the mean flux; only the frictional and eddy fluxes can affect the total. At the streamline coinciding with the basin boundary only fric- tional fluxes can act to ultimately remove any net vorticity input as $0=\mathbf{u}^{\prime} \cdot \hat{\mathbf{n}}$ at the boundary.

To achieve (5), either frictional fluxes or eddy fluxes must act prominently somewhere along every streamline enclosing a net source of vorticity (as noted by Niiler 1966). The nondimensional parameters anticipate which fluxes are expected to be important in this process; the frictional widths, $\delta_{M}$ and $\delta_{S}$, can be compared to the inertial width, $\delta_{I}$. A boundary layer Reynolds number $\left(\mathrm{Re} \equiv \delta_{I}^{3} / \delta_{M}^{3}\right)$ is useful in this comparison. $^{2}$

When the Reynolds number is less than 1 , frictional effects are expected to dominate in the boundary current. For example, in the Munk (1950) problem where $\operatorname{Re}=0$, a $\beta$ flux receives the wind vorticity input [Sverdrup (1947) balance] and transports it westward to the boundary current. There it is exchanged to a frictional flux and removed from the basin. If $0<\operatorname{Re} \leq 1$, then the $\beta$ flux and mean flux combine to transport the wind vorticity input to the frictional region of a western boundary current, in that case a visco-inertial boundary current.

Even though eddies may be present, eddy vorticity fluxes are weak when $\operatorname{Re} \leq O(1)$. In this regime, many useful studies exist of steady solutions (Moro 1987, 1988; Cessi and Ierley 1990; Ierley and Sheremet 1995; Speich et al. 1995; Primeau 1998) and the onset of time dependence through unstable perturbations about the mean (Moro 1990; Speich et al. 1995; Sheremet et al. 1997; Dijkstra and Katsman 1997; van der Vaart et al. 2002). Low-frequency variability onset is particularly interesting (e.g., Berloff and Meacham 1998; Berloff 1998; Nauw and Dijkstra 2001; Chang et al. 2001) and is related to the onset of gyre symmetry-breaking modes, a subject that will be important here. However, since these analyses begin with a steady solution where eddy fluxes cannot contribute, only the existence of eddies can be studied, not the effects of eddy fluxes on the equilibrium flow.

The focus here is on Reynolds number greater than 1 , where inertial and eddy effects are expected to dominate. The boundary layer Reynolds number relevant for oceanic flows is likely to be $O(10-1000) .^{3}$ In this regime, Il'in and Kamenkovich (1964) and Ierley and Ruehr (1986) demonstrate that a steady western boundary current cannot exist. Heuristically, the sea-

\footnotetext{
${ }^{2}$ Other authors use a basin-dimension Reynolds number that is a factor of $L / \delta_{I}$ larger.

${ }^{3}$ The Reynolds number depends on what the model friction represents, e.g., eddy viscosity or proxy for bottom topography, and is best taken as a model-specific quantity rather than a measurable quantity.
} 
ward portion of the visco-inertial boundary current does not enter the frictional sublayer and thus cannot dissipate enough relative vorticity. Thus, in this highly nonlinear regime, equilibrium occurs by 1) the current becoming unsteady (Edwards and Pedlosky 1998; FoxKemper and Pedlosky 2004), 2) formation of a recirculation gyre (Ierley 1987), or 3) vorticity exchange with an opposing wind forcing region (Harrison and Holland 1981; Marshall 1984). In case 1, eddy fluxes transport vorticity across closed interior streamlines to a frictional region nearer the boundary, thus eddy fluxes satisfy (5) for interior streamlines and frictional fluxes do so for near-boundary streamlines. This process will be called an eddy flux to the frictional sublayer, and results in a boundary current where eddy fluxes are important at first order (Fox-Kemper 2004). In case 2, the streamlines are rearranged-which also affects the frictional and eddy fluxes-and thereby (5) is satisfied. For example, contact of the recirculation gyre with the eastern boundary radically changes the source of frictional vorticity and the eddy fluxes (Fox-Kemper and Pedlosky 2004). In case 3 , vorticity is transported from a region of vorticity input to a region with opposing input, and thus the net burden is reduced. This reduction can occur either by intergyre mean flux, where the mean streamlines move to enclose opposing wind input, thus reducing the right-hand side of (5), or by an intergyre eddy flux, where eddies flux vorticity across mean streamlines (Harrison and Holland 1981; Marshall 1984). An intergyre eddy flux does not require a permanent exchange of fluid; Lozier and Riser (1990) propose that dissipative meandering also occurs, where fluid is temporarily exchanged and acted on by dissipation before returning.

A large feedback from eddies to the mean flow requires determination of the eddy fluxes to find the mean flow, but the eddy fluxes prove rather sensitive and subtle, even in a barotropic model. Thus for simplicity, baroclinic and topographic effects are ignored here, although they are undoubtedly significant (see Ghil et al. 2002; Berloff 2005; Marshall and Stephens 1998; Griffiths and Veronis 1997; Griffiths 1998; Becker and Salmon 1997; Hughes and de Cuevas 2001). The simplicity of the models used here allows for careful, well-resolved study of a higher and wider range of Reynolds numbers than is possible in more inclusive models. The more extensive analysis of the barotropic model readily extends to understanding more complex models.

Though simple, the barotropic model has rich eddy dynamics. Eddy fluxes are often assumed to behave similarly to frictional or "eddy viscosity" fluxes, mixing more where mean gradients are largest. Yet in this model, the location of the largest gradients in the mean vorticity do not coincide with the largest eddy fluxes because the largest gradients act as barriers to eddy transport (Bower and Lozier 1994; Rogerson et al. 1999; Yuan et al. 2004). Heuristically, eddy viscosity treats eddy mixing as an analog to the mixing by molecule movement. However, molecules are similar to each other and have only limited interaction, while eddies have structure and interaction with each other and the mean flow related to their origin as an instability of the mean flow. Here, eddy structure and interaction are demonstrated to have a pronounced effect on the eddy fluxes. Also, the diagnostic separation of the flow into "eddy" and "mean" contributions understates the potential interchange of the eddy and mean fluxes (a good discussion on dynamically based categorization is Berloff 2005). As the model obeys (1) not (3), the model need not recognize the averaging done in postintegration calculation of (3). It is demonstrated that with a change of boundary conditions or gyre symmetry the role played by the eddy fluxes may go to the mean fluxes. Thus resolved eddies have a rather different response to parameter changes than frictional or eddy viscosity fluxes.

Much of the action of eddy fluxes can be diagnosed with an eddy efficiency. Eddies are both formed by and destroyers of shear, so what equilibrium is struck is a function of how efficient the eddies are at mixing away the shear versus how unstable the shear is. A quantitative measure of eddy efficiency can be constructed by comparing the eddy fluxes to the frictional fluxes, similar to the Nusselt number of thermal convection. The measure is defined as a ratio of integrals of fluxes across a mean streamline $\left(\psi_{c}\right)$ :

$$
\mathrm{Nu}_{\zeta}\left(\psi_{c}\right) \equiv \frac{\oint_{\psi_{c}}\left(\delta_{I}^{2} \overline{\mathbf{u}^{\prime} \zeta^{\prime}}+\delta_{S} \nabla \bar{\psi}-\delta_{M}^{3} \nabla \bar{\zeta}\right) \cdot \hat{\mathbf{n}} d s}{\oint_{\psi_{c}}\left(\delta_{S} \nabla \bar{\psi}-\delta_{M}^{3} \nabla \bar{\zeta}\right) \cdot \hat{\mathbf{n}} d s} .
$$

These integrals are calculated easily and accurately as area integrals of the flux divergence (using Gauss's theorem) and, if more convenient, $\overline{\mathbf{u} \zeta}$ may be substituted for $\overline{\mathbf{u}^{\prime} \zeta^{\prime}}$ since the $\overline{\mathbf{u}} \bar{\zeta}$ contribution vanishes due to the region of integration:

$$
\mathrm{Nu}_{\bar{\zeta}}\left(\psi_{c}\right) \equiv \frac{\int_{\bar{\psi} \geq \psi_{c}} \nabla \cdot\left(\delta_{I}^{2} \overline{\mathbf{u} \zeta}+\delta_{S} \nabla \bar{\psi}-\delta_{M}^{3} \nabla \bar{\zeta}\right) d A}{\iint_{\bar{\psi} \geq \psi_{c}} \nabla \cdot\left(\delta_{S} \nabla \bar{\psi}-\delta_{M}^{3} \nabla \bar{\zeta}\right) d A} .
$$


This definition naturally and uniquely selects for the divergent eddy fluxes and ignores the rotational fluxes (Fox-Kemper et al. 2003). For each mean streamline, $\mathrm{Nu}_{\zeta}$ compares the total amount of cross-streamline vorticity flux to the purely frictional flux. ${ }^{4}$ If the eddies are inconsequential, this number will be one. The more efficient the eddies are at fluxing vorticity across streamlines and reducing vorticity gradients, the larger $\mathrm{Nu}_{\zeta}$ becomes. If the eddies are working upgradient (against the friction), then $\mathrm{Nu}_{\zeta}$ is less than one. More discussion of cross-streamline fluxes and $\mathrm{Nu}_{\zeta}$ can be found in FoxKemper and Pedlosky (2004).

The following section provides details about the numerical model and forcing used. The next section presents and analyzes the numerical results of the calculation and is broken into thematic subsections comparing different sensitivities of the eddy fluxes. The final section summarizes and concludes.

\section{Models}

As in Fox-Kemper and Pedlosky (2004), a Chebyshev polynomial pseudospectral numerical barotropic model is used in a $\beta$-plane rectangular basin. A single constantdepth layer is assumed, so the flow is nondivergent and only the vorticity equation is solved. The nondimensional equations governing the model are (1) and (2).

The calculations are differentiated by the values of the constant parameters $\left(\delta_{I}, \delta_{S}, \delta_{M}\right)$, as well as the initial conditions, frictional boundary conditions on the "solid" boundaries, and the wind forcing. The initial conditions are usually resting $(\psi=0)$, except for the double-gyre calculations. Most of the double-gyre calculations are not started from a symmetry-breaking initial condition, which reduces the spinup time for reasons discussed in section 3c. The boundary conditions are slip $(\zeta=0)$ on the northern and southern "fluid" boundaries and either slip or no slip $(\partial \psi / \partial x=0)$ on the eastern and western solid boundaries. Impermeability is imposed on all boundaries $(\psi=0)$. For most calculations $\delta_{I}=0.02$ and $\delta_{S}=0$, except for the calculations in Figs. 13 and $14 .^{5}$

Three different wind forcings $\left(w_{E}\right)$ are used, each with a corresponding basin dimension. These forcings and basins are constructed so that the subtropical gyre is relatively unchanged as the subpolar gyre changes. The single gyre has only subtropical forcing:

\footnotetext{
${ }^{4}$ Note that $\mathrm{Nu}_{\zeta}$ is a function of the mean streamline across which it is calculated.

${ }^{5}$ The calculations in Fig. 14 were unresolvable with $\delta_{I}=0.02$ and available computers, hence the increase to $\delta_{I}=0.06$ for those calculations.
}

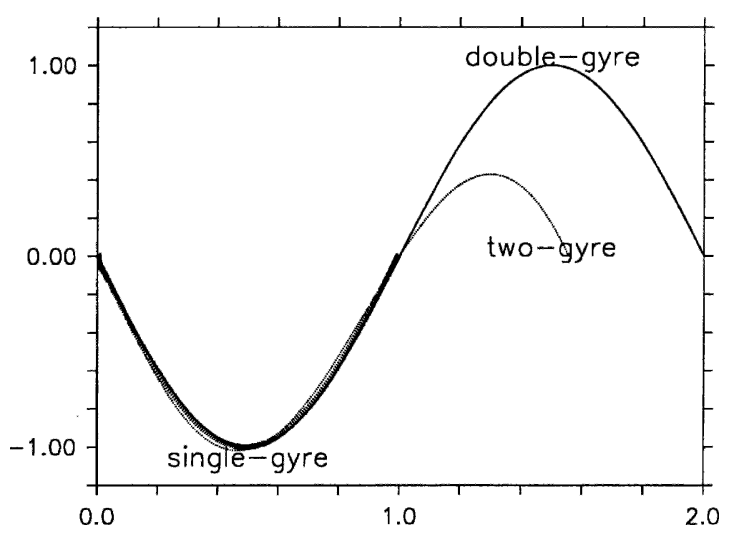

FIG. 1. Wind vorticity input, $w_{E}$, in the three models.

$$
w_{E}=-\sin (\pi y), \quad \forall: 0<x<1,0<y<1 .
$$

The double gyre has antisymmetric subtropical and subpolar forcing:

$$
w_{E}=-\sin (\pi y), \quad \forall: 0<x<1,0<y<2 .
$$

The double-gyre forcing can be a misleading representation of the ocean, as the two gyres are exactly antisymmetric. The two-gyre forcing is a polynomial approximation to smoothly combine the standard subtropical wind forcing to a smaller, weaker subpolar forcing than in the double-gyre forcing. The two-gyre $w_{E}$ in (10) guarantees that the integrated vorticity input rate to the subtropical gyre is $2 / \pi$ as in the single- and double-gyre forcings and that $w_{E}=0$ at the southern boundary, at $y=1$, and at the northern boundary. The relative area and vorticity input to each gyre in the two-gyre model are matched approximately to Pacific Ocean conditions. The expression for the two-gyre $w_{E}$ is

$$
\begin{aligned}
& w_{E}=-\frac{2}{\pi}\left(1+\frac{4 y}{L_{y}}\right) \frac{\frac{5 y^{4}}{L_{y}^{4}}-\frac{16 y^{3}}{L_{y}^{3}}+\frac{16 y^{2}}{L_{y}^{2}}-\frac{5 y}{L_{y}}}{\frac{10}{3 L_{y}^{5}}-\frac{59}{5 L_{y}^{4}}+\frac{12}{L_{y}^{3}}-\frac{4}{3 L_{y}^{2}}-\frac{5}{2 L_{y}}}, \\
& L_{y} \equiv \frac{11+\sqrt{21}}{10} \approx 1.56, \quad \forall: 0<x<1,0<y<L_{y} .
\end{aligned}
$$

The three forcings are shown in Fig. 1.

Sufficient resolution is assured by the spectral decay of relative vorticity and by comparison of calculations at different resolutions. Typical resolutions are $257 \times$ 257 modes for single gyre and $513 \times 257$ for double gyre and two-gyre (although $769 \times 257$ was required for some calculations). The calculations involving bottom friction were run at marginal $(257 \times 129)$ resolution, but the additional damping from the bottom friction provides fair accuracy. It should be noted that the ir- 

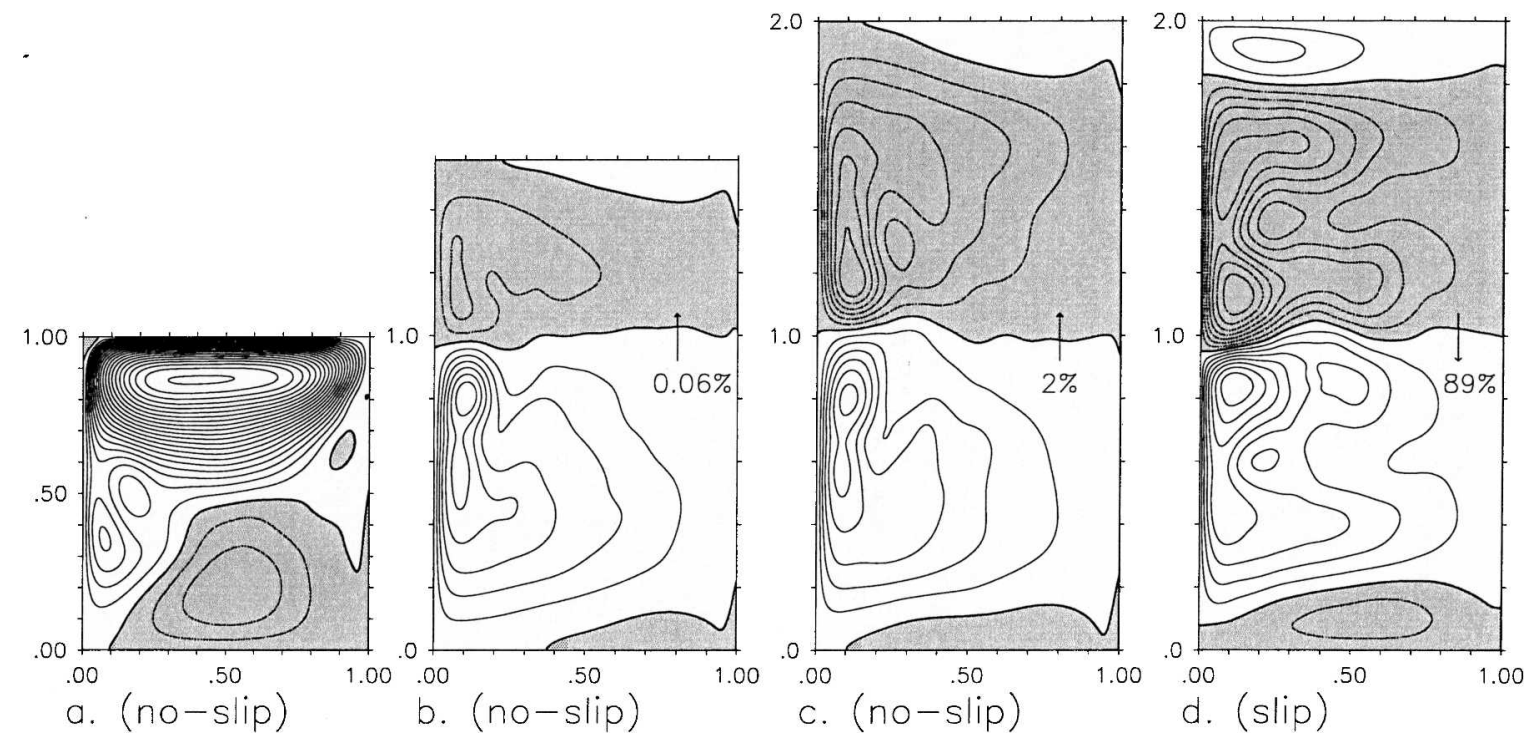

FIG. 2. Contours of the time-mean streamfunction for different basins, wind forcing, and boundary conditions with $\mathrm{Re}=5$ : (a) no-slip single-gyre, (b) no-slip two-gyre, (c) no-slip double-gyre, and (d) slip double-gyre model. The wind input to the subtropical gyre is $2 / \pi$ in all the calculations. The labeled arrows on the figure give the eddy vorticity flux as a percentage of the subtropical wind input across the zero streamfunction contour dividing the gyres. The contour interval is 0.2 , and shading indicates negative streamfunction.

regular Chebyshev collocation grid provides much higher resolution in the boundary current: ten times more "grid points" than is common in finite difference calculations.

\section{Numerical results}

\section{a. The effect of boundary conditions on intergyre vorticity flux}

Surprisingly, the frictional boundary conditions exert a controlling influence on the amount of intergyre eddy vorticity flux. Figure 2 shows that there is almost no intergyre eddy vorticity flux with no-slip boundary conditions. Double-gyre calculations with slip boundary conditions result in the more traditional finding - that there is a large intergyre eddy flux. The eddy flux across $y=1$ is shown in Fig. 3 .

Both dissipative meandering and Lagrangian fluid exchange contribute to the intergyre eddy flux in the slip double gyre. Snapshots of the relative vorticity evolution where the boundary currents separate are shown in Fig. 4. At time $t=5035$, the subpolar western boundary current overshoots across the mean streamline denoting the gyre boundary (the gyre-dividing streamline), bringing anomalous positive relative vorticity into the mean subtropical gyre. This intrusion produces a negative vorticity flux. Note how the frictional flux anomaly shown next to each snapshot reflects the overshooting boundary current. By $t=5070$, the subpolar vorticity anomaly left the boundary, partly by the anomalous dissipation through the western boundary (dissipative meandering), and the remainder has moved into the basin interior. Note that the core of this subpolar eddy does not cross permanently into the subtropical gyre; it returns to the subpolar gyre.

At $t=5075$, some subtropical water intrudes into the subpolar gyre. Because the subtropical water has anomalous negative vorticity, the eddy flux across the gyre boundary is negative, as for the subpolar over-

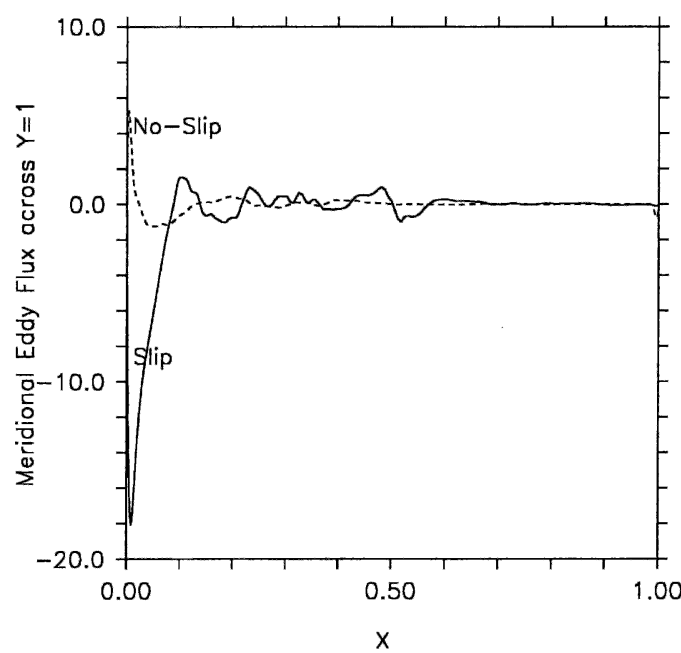

FIG. 3. Direct estimates of eddy vorticity fluxes across $y=1$ for $\mathrm{Re}=5$ double-gyre calculations with different boundary conditions. (The mean fluxes across $y=1$ are negligibly small in comparison.) 

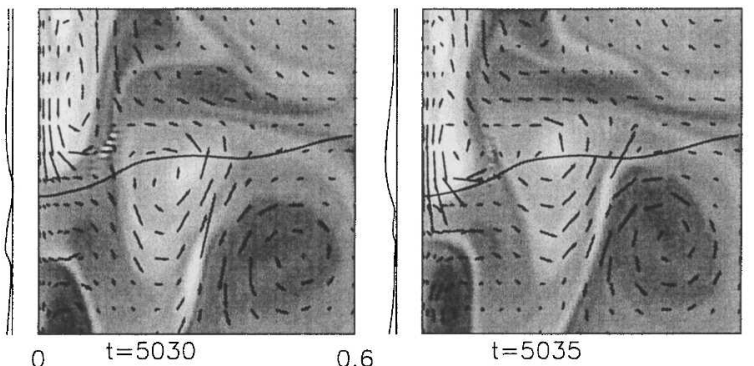

$t=5035$

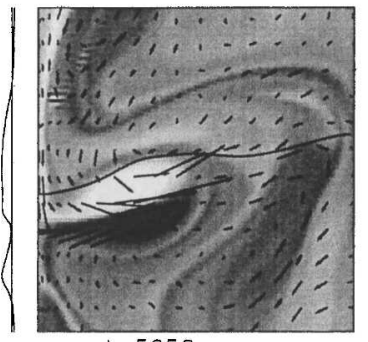

$\mathrm{t}=5050$

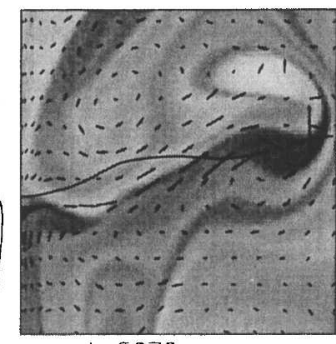

$\mathrm{t}=5070$

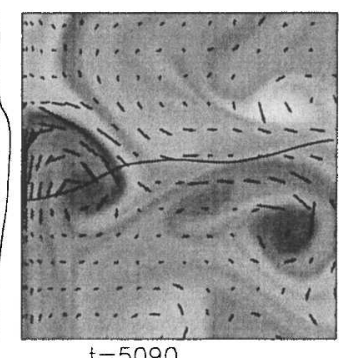

$t=5090$

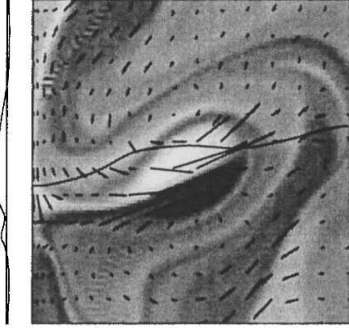

$t=5055$

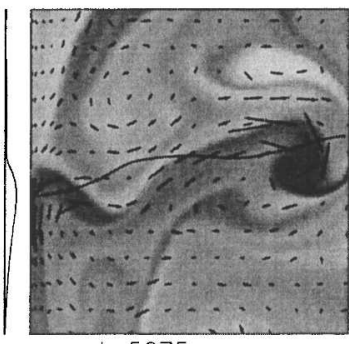

$t=5075$

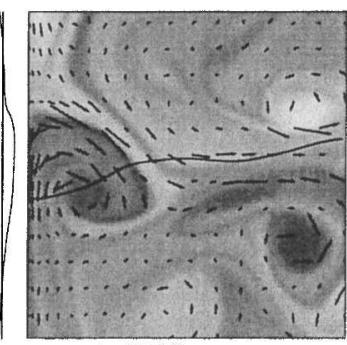

$t=5095$

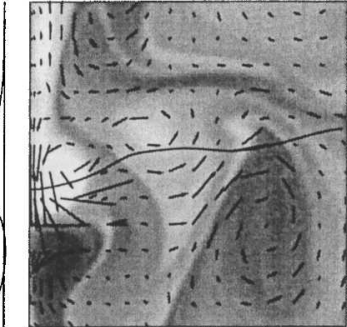

$t=5040$

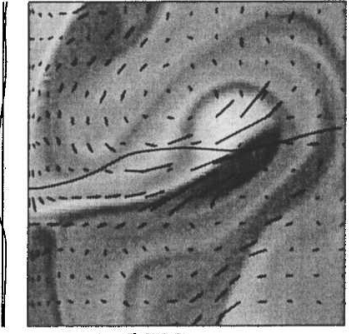

$t=5060$

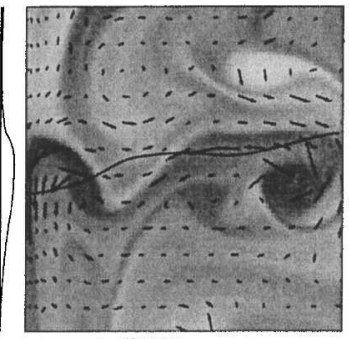

$t=5080$

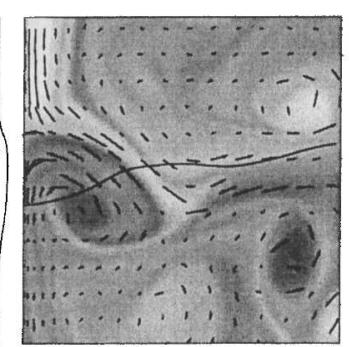

$t=5100$

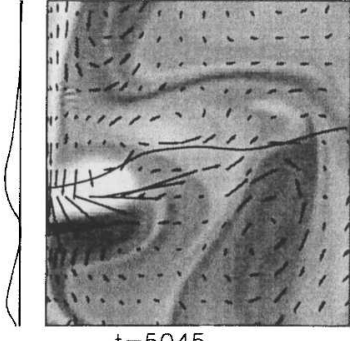

$t=5045$

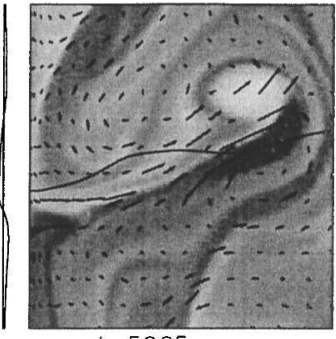

$t=5065$

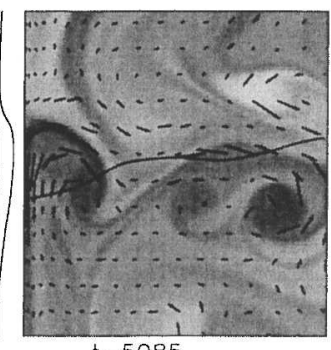

$t=5085$

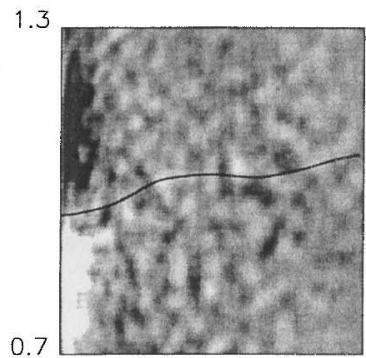

OEddy Flux Divergence 0.6

FIG. 4. Snapshots of the separation region of jet from the coast $(0<x<0.6,0.7<y<1.3)$ in the slip, double-gyre Re $=5$ calculation. Relative vorticity is shaded, darker is more negative, and lighter is more positive. The solid line denotes the average position of the intergyre boundary streamline. Arrows denote the local instantaneous value of eddy vorticity flux, $\mathbf{u}^{\prime} \zeta^{\prime}$. The line plot to the left of each snapshot shows the anomalous frictional flux through the western boundary as a function of $y$. The lower right image shades the time-mean eddy flux divergence with dark (light) for divergences (convergences).

shoot at $t=5035$. The frictional flux anomaly again reveals dissipative meandering. The last image in Fig. 4 shows that the eddy flux divergences agree with this analysis of the intergyre eddy fluxes.

Significant dissipative meandering must occur near the western boundary. A vorticity anomaly displaced across the gyre-dividing streamline in the interior will propagate as a Rossby wave packet and only dissipate mildly. However, as shown in Fig. 4, if the anomaly is near the boundary, significant dissipation can occur via a frictional flux through the boundary before the anomaly returns to its native gyre.

If it is assumed that the majority of dissipative meandering is at the boundary, a lower estimate of the portion of the intergyre eddy flux due to dissipative meandering may be calculated. The frictional flux perturbations shown in Fig. 4 are sometimes many times larger at a given point than the mean frictional flux for a given snapshot, but isolated in space near the gyredividing jet. When only the frictional perturbations that 
time-mean meridional velocity
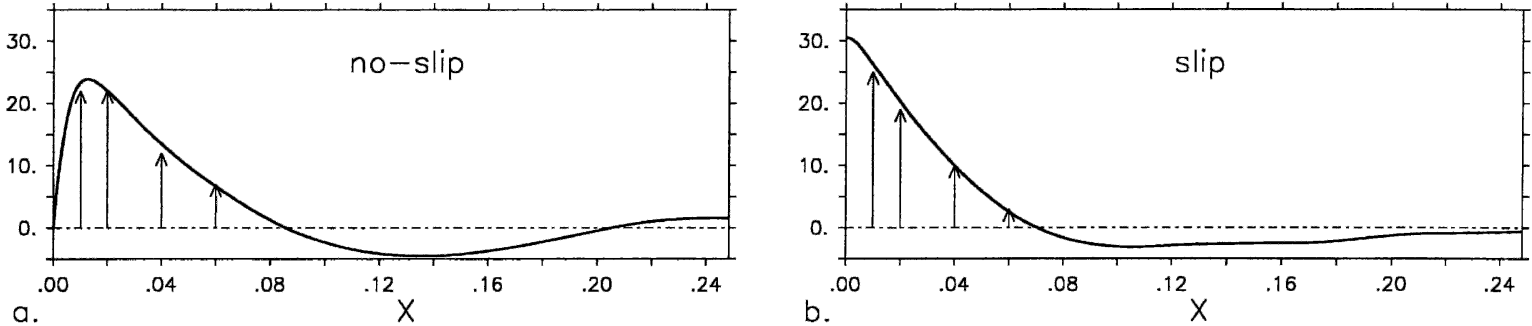

time-mean rel. vort
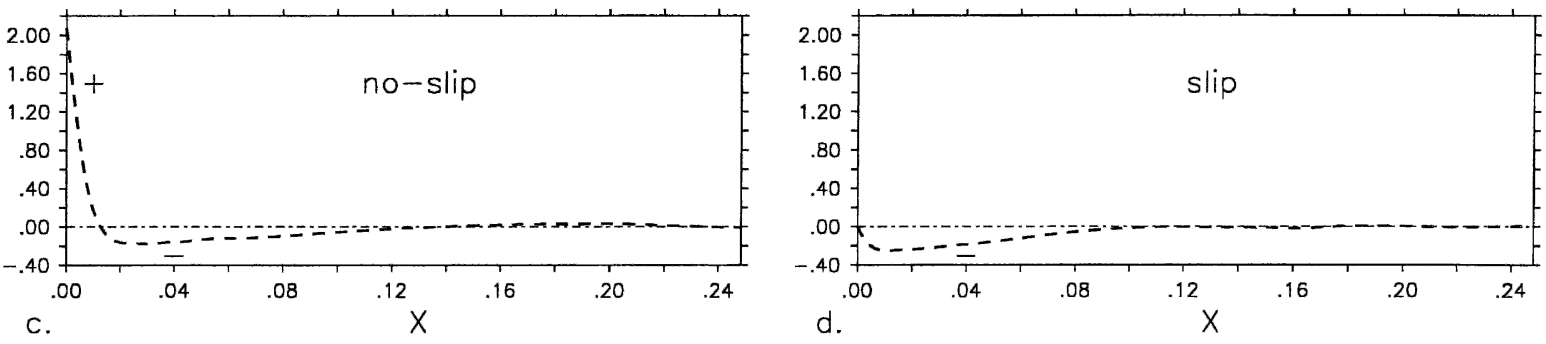

FIG. 5. (a), (b) Meridional velocity and (c), (d) relative vorticity near the boundary layer at $y=0.5$ in the subtropical gyre for (a), (c) no slip and (b), (d) slip calculations.

are of different sign than the mean are considered (i.e., those associated with vorticity anomalies from the other gyre) and they are integrated along the boundary of a gyre and averaged in time, 2.6 times as much vorticity is fluxed through the boundary by dissipative meandering perturbations as by the mean frictional flux for the calculation shown in Fig. 4. Since the intergyre eddy flux including the dissipative meanders is $89 \%$ of the total vorticity input to the subtropical gyre, this indicates that approximately $28 \%$ of the total is due to dissipative meandering.

As noted by Berloff et al. (2002), the initial crossing of fluid parcels that are ultimately exchanged also occurs primarily near the western boundary. Once a fluid parcel crosses the gyre-dividing streamline in the western boundary current, it is usually swept in the jet to the east and often they return to their native gyre as in Fig. 4. Occasionally, they end up crossing permanently into the other gyre at the eastern end of the jet. A passive tracer study (as in Berloff et al. 2002) or a lobe dynamics study (as in Coulliette and Wiggins 2001) is needed to determine how often this occurs and is beyond the scope here, but the results of Berloff et al. (2002) suggest that $O(10 \%)$ of the trajectories switch gyres at this Reynolds number [slightly larger than the largest Berloff et al. (2002) used].

Both intergyre eddy flux mechanisms thus depend critically on alternating overshooting of the boundary current. The weak vorticity flux away from the boundary in Fig. 3 reflects this dependence.
The intergyre eddy flux in the no-slip calculations differs from that in the slip calculations for two reasons (see Fig. 5). 1) Boundary current overshoot is reduced due to the zero tangential velocity at the wall in the no-slip calculations. Thus, the maximum magnitude of no-slip vorticity flux is smaller than the slip maximum in Fig. 3. 2) Even when an overshoot or eddy shedding does occur from a no-slip boundary current, the relative vorticity is not sign-definite as in the slip boundary current (Fig. 5c versus Fig. 5d). As Stewart (1964, 1989) points out, a no-slip boundary current under many circumstances transports no net vorticity because it advects both positive and negative relative vorticity (Fig. $5 \mathrm{c})$. The vortices shed from the boundary current keep approximately the vorticity distribution of the current, thus a dipole of eddies typically results from the instability of the boundary current. If the dipole makes its way into the other gyre, the vorticity advected tends to cancel, which explains the positive-peak, negative-peak shape of the no-slip flux in Fig. 3. The details of each eddy shedding event determine if there is any difference between the two vortices in the pair, for example, by viscous interactions with the boundary. The intergyre eddy flux in no-slip calculations typically slightly increases the amount of vorticity friction must remove (e.g., Figs. 2b,c).

Thus, the boundary conditions strongly affect the structure of the boundary current through their effect on the structure of the eddies formed. Intergyre eddy flux, by dissipative meandering or fluid parcel ex- 

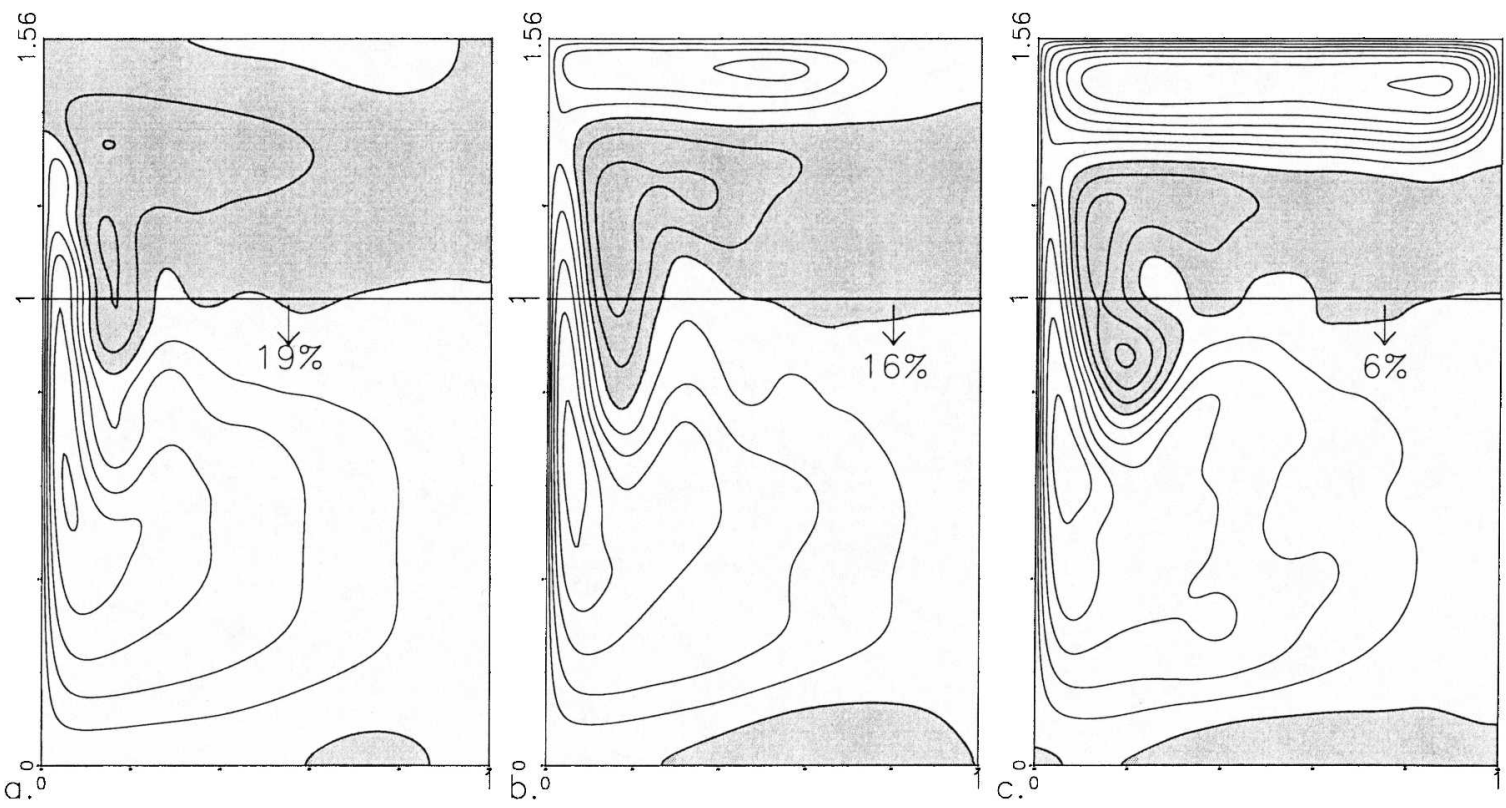

FIG. 6. Comparison of two-gyre slip calculations with increasing Reynolds number. The arrow indicates the eddy flux across the mean streamline dividing the gyres as a percentage of the wind input in the $0<x<1,0<y<1$ region: (a) $\operatorname{Re}=2.5$, (b) $\operatorname{Re}=5$, and (c) $\operatorname{Re}=10$.

change, seems limited to slippery boundary conditions and occurs primarily in a narrow region near the boundary.

\section{b. Effects of gyre symmetry}

The previous subsection shows that intergyre eddy flux results from alternating overshooting of the boundary current into the opposing gyre. This section explores the role of the relative strength of the gyres in this overshooting process.

The double-gyre wind forcing can be misleading as a representation of the ocean because, unlike the oceans, it has exactly antisymmetric vorticity input. In the ocean, the region occupied by the subpolar gyre is significantly smaller, and the wind stress curl is also smaller there. Thus the circulation input to the subpolar gyre should be much weaker.

The two-gyre wind forcing is designed to study the effects of gyre asymmetry. At low Reynolds number, there is little difference in the dynamics of the two-gyre and double-gyre calculations. The steady solutions in both forcing/basin configurations exhibit separation near the zero wind stress curl line, and the removal of vorticity is frictional.

However, at higher Reynolds number, there is a significant change in the structure of the slip two-gyre and double-gyre models - the boundary current from the stronger, subtropical gyre in the two-gyre model permanently overshoots (Fig. 6). The intergyre eddy flux in the double-gyre solution requires alternation between a subtropical and subpolar boundary current overshoot, but this strict alternation is a singular case restricted to the antisymmetric double-gyre model.

Intergyre mean flow appears to be the preferred mechanism for sharing vorticity between the gyres in slip calculations at high Reynolds number. As Fig. 6 reveals, the amount of eddy intergyre vorticity flux decreases and the mean flux increases as the Reynolds number increases. It seems that at sufficiently high Reynolds number, the recirculation of the stronger gyre would take over the weaker gyre, leaving only one basin-filling gyre. This gyre would only require friction to balance the net circulation input, as wind forcing of both signs would contribute to the right-hand side of (5). Figure $7 \mathrm{~b}$ shows the mean streamfunction in a slip calculation with no subpolar wind forcing. By comparing with Fig. 6b, it is clear that the mean flow from the subtropical gyre is strongly reduced by its exposure to the subpolar wind forcing in Fig. $6 \mathrm{~b}$.

The no-slip, two-gyre calculation (Fig. 2b) does not permanently overshoot. Cessi (1991) shows that, when boundary currents of unequal strength meet, the degree of overshoot depends strongly on the boundary conditions. For slip conditions, the stronger current overshoots, while for no-slip conditions, the stronger current undershoots (to a lesser degree). Haidvogel et al. (1992) also found this effect in a model that smoothly adjusted from slip to no-slip boundary conditions. The 

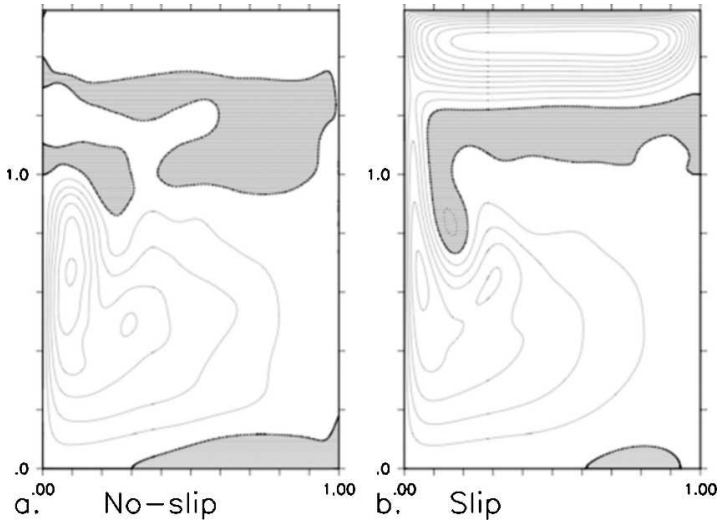

FIG. 7. Time-mean streamfunction for $\mathrm{Re}=5$ calculations with single-gyre wind forcing between $y=0$ and 1 and no wind forcing for $y>1$. The basin dimension is the same as in the two-gyre calculations. The contour interval is 0.2 , and shading indicates negative streamfunction.

analysis of Cessi (1991) gives a local explanation for the difference between the two-gyre no-slip and slip calculations (Fig. 2b versus Fig. 6b).

The eddy intergyre vorticity flux is critically important to one remaining region in the slip two-gyre model. The remaining subpolar gyre is cut off from the western boundary, so cannot dissipate its vorticity with a mean western boundary current. Thus, an eddy flux out of the subpolar gyre is required and is nearly equal to the wind input within that gyre. This eddy flux occurs primarily by interruptions of the subtropical western boundary current overshoot with subpolar waters.

In summary, in slip calculations with asymmetric gyres, the preferred mechanism for interchange between the gyres is by an Eulerian mean overshoot of the stronger gyre boundary current (which averages a rather unsteady current due to interruptions by the weaker gyre waters). No-slip calculations do not overshoot, at least in this range of Reynolds numbers. Thus, the alternating boundary current overshoot resulting in intergyre eddy fluxes is limited to only the singular case of the antisymmetric double gyre at Reynolds numbers much greater than 1 .

\section{c. Effects of eddy symmetry and efficiency}

The first surprising result of the no-slip calculations shown in Fig. 2 is that the intergyre eddy vorticity fluxes are so small. The second, perhaps more surprising result, is that despite the lack of intergyre eddy fluxes, the circulation strength of each gyre is still greatly reduced in the two-gyre and double-gyre calculations compared to the single-gyre case. Figure $7 \mathrm{a}$ shows that the reduced circulation is not due to internal cancellation of vorticity, as no subpolar wind forcing is required to reduce the circulation! The preceding subsection demonstrates that the symmetry of the gyres about the jet is important. This section demonstrates that the symmetry of the eddies is also important through its effect on the eddy efficiency.

Eigenmodes of instability on a jet are usually categorized by symmetry across the jet. The sinuous modes are even in streamfunction perturbation about the jet center and therefore produce a wiggling, meandering advection pattern (Fig. 8). The varicose modes are odd in streamfunction perturbation and therefore produce mirror-reflection-symmetric, knotted advection pattern about the jet center (Fig. 8).

A solid, slip boundary condition is similar to a mirror reflection about the boundary. Thus, analogues of varicose modes are free to form in the extension of the western boundary current along the northern boundary in a single-gyre model with northern boundary slip conditions. The formation of sinuous modes in the singlegyre model is prohibited, as the sinuous modes cause the jet to meander but the solid boundary cannot meander. The critical difference between the no-slip single-gyre and multiple-gyre calculations is not an intergyre eddy flux of vorticity, but the arrival of new modes of instability on the jet.

It is well known that sinuous modes typically have larger growth rates and lead to more mixing than the varicose modes. Balmforth and Piccolo (2001) present an excellent analysis of eigenmodes and their mixing in a zonal jet obeying equivalent dynamical equations to (1). Their results indicate that the sinuous mode growth is much faster than the varicose mode growth and that sinuous modes induce strong mixing to either side of
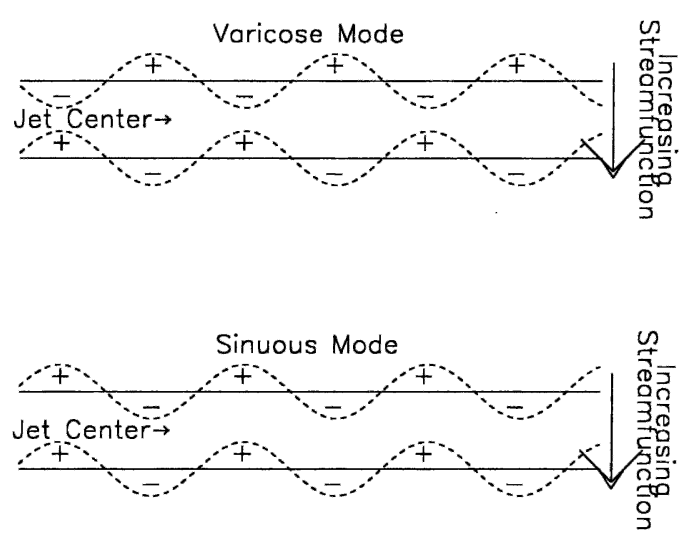

FIG. 8. Schematic of sinuous and varicose modes of instability on a jet. The solid lines represent initial jet streamlines, the dashed lines represent streamlines after the instability has grown somewhat. The pluses and minuses denote streamfunction anomalies. 


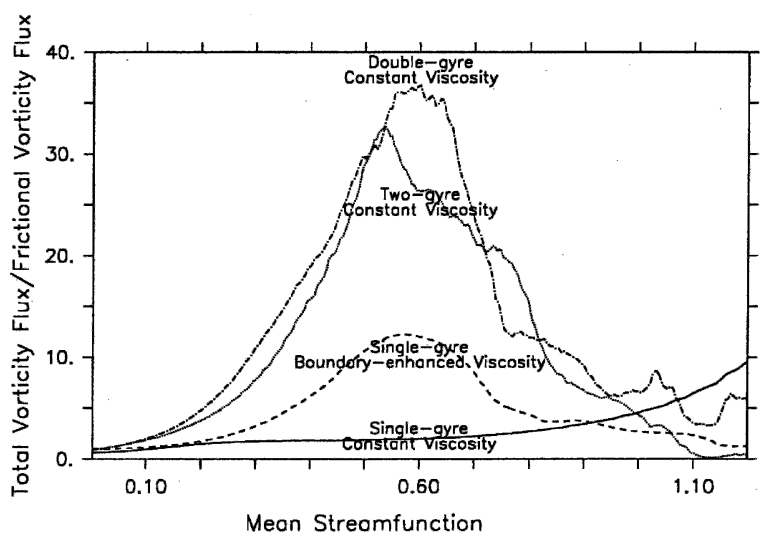

FIG. 9. The ratio $\mathrm{Nu}_{\zeta}$ as a function of mean streamfunction. The ratio is shown for the no-slip $\mathrm{Re}=5$ calculations (single, double, and two-gyre) and the $\mathrm{Re}_{b}=0.25, \mathrm{Re}_{i}=5$ no-slip, single-gyre calculation. See (11) for a definition of $\mathrm{Re}_{b}$ and $\mathrm{Re}_{i}$.

the jet, although not in the jet core. Numerical simulations find similar results (e.g., Pratt et al. 1995; Rogerson et al. 1999).

The difference between the single gyre and the double gyre is in the eddy efficiency, $\mathrm{Nu}_{\zeta}$. The sinuous modes do not flux vorticity between gyres in no-slip calculations, so the eddy flux can only be to the frictional sublayer of the same gyre, just as in the single gyre. Thus, the eddy flux sources and sinks are identical in the multiple-gyre and the single-gyre cases. Figure 9 shows that the double-gyre and two-gyre calculations have a $\mathrm{Nu}_{\zeta}$ for interior streamlines (approximately $\left.0.2<\psi_{c}<1\right)$ that indicates that the combination of sinuous and varicose eddies in these multiple-gyre calculations is 30 times more efficient at fluxing vorticity in the basin interior than the friction alone. The combination of sinuous and variance modes in the multiplegyre case leads to a $\mathrm{Nu}_{\zeta} 15$ times the $\mathrm{Nu}_{\zeta}$ in the singlegyre calculation, which is the mixing efficiency of varicose eddies alone (Table 1, columns for Figs. $2 \mathrm{a}$ and 2b).
Figure 10 depicts a numerical demonstration of the effects of sinuous modes. Just as sinuous modes are prevented in the single gyre, they are also prevented by the initial symmetry about $y=1$ of double-gyre calculation started from rest. Two double-gyre calculations that differ only in initial conditions are shown in Fig. 10. One is started with symmetry-breaking initial conditions, rapidly develops sinuous modes, and soon equilibrates about a mean (Fig. 10d). The one started from rest has no initial infinitesimal sinuous perturbations, so it first spins up to a near equilibrium strongly resembling the single-gyre calculation (Fig. 10a versus Fig. 10b). Eventually, small numerical errors break the gyre symmetry, ${ }^{6}$ and the sinuous modes grow rapidly. Once the excess vorticity buildup has been removed by friction, a new equilibrium is reached (Fig. 10c) that resembles that of the model begun with symmetrybreaking initial conditions (Fig. 10d).

Varicose modes and eddies, the growth of sinuous modes and eddies, and their effects on the jet and recirculations are shown in Fig. 11. The upper row of this figure shows snapshots from before the symmetry between the gyres breaks (as in Fig. 10a). The recirculation gyre and gyre-dividing jet cross the entire basin, despite the fact that there are a number of strong eddies formed by varicose instabilities. The second, third, and fourth rows show the progress of the sinuous modes and eddies soon after the antisymmetry is broken. The gyre-dividing jet shortens, and many eddies pierce the recirculation region. The bottom row shows a typical series of snapshots after equilibration with sinuous modes

\footnotetext{
${ }^{6}$ The duration of model runs before the numerics break the symmetry is a strong function of resolution. The calculation in Fig. 10a has $513 \times 257$ modes, which is close to the minimum needed to arrive at a near equilibrium resembling the single gyre $(401 \times$ 257 is suggestive but insufficient). The initial conditions make little difference at lower resolutions, as symmetry is immediately broken by truncation errors.
}

TABLE 1. Values of $\mathrm{Nu}_{\zeta}\left(\psi_{\mathrm{c}}=0.5\right)$ and percentage of subtropical wind input removed from the subtropical gyre by intergyre eddy, lateral friction, and bottom friction fluxes for various calculations. A typical mean streamline, $\psi_{c}=0.5$ (halfway between 0 and the linear Munk solution maximum), is used. Percentages may not add up to $100 \%$ because fluxes are independently determined and contain small averaging errors.

\begin{tabular}{|c|c|c|c|c|c|c|c|c|c|c|}
\hline & \multicolumn{10}{|c|}{ Figure } \\
\hline & $2 \mathrm{a}$ & $2 b$ & $2 \mathrm{c}$ & $2 \mathrm{~d}$ & $13 \mathrm{a}$ & $13 b$ & $13 \mathrm{c}$ & $13 \mathrm{~d}$ & $13 \mathrm{e}$ & $13 f$ \\
\hline Boundary condition & No slip & No slip & No slip & Slip & Slip & No slip & Slip & No slip & Slip & No slip \\
\hline $\mathrm{Re}$ & 5 & 5 & 5 & 5 & 5 & 5 & 5 & 5 & 25 & 25 \\
\hline$\delta_{S}$ & 0 & 0 & 0 & 0 & 0.001 & 0.001 & 0.003 & 0.003 & 0.005 & 0.005 \\
\hline $\mathrm{Nu}_{\zeta}\left(\psi_{c}=0.5\right)$ & 1.9 & 29 & 29 & 28 & 3.3 & 3.2 & 1.3 & 1.1 & 1.4 & 2.3 \\
\hline Intergyre eddy & 0 & $-0.06 \%$ & $-2 \%$ & $89 \%$ & $56 \%$ & $-1 \%$ & $16 \%$ & $-1 \%$ & $30 \%$ & $-2 \%$ \\
\hline Bottom friction & $0 \%$ & $0 \%$ & $0 \%$ & $0 \%$ & $6 \%$ & $1 \%$ & $31 \%$ & $10 \%$ & $41 \%$ & $7 \%$ \\
\hline Lateral friction & $100 \%$ & $100 \%$ & $101 \%$ & $11 \%$ & $39 \%$ & $100 \%$ & $55 \%$ & $92 \%$ & $28 \%$ & $94 \%$ \\
\hline
\end{tabular}




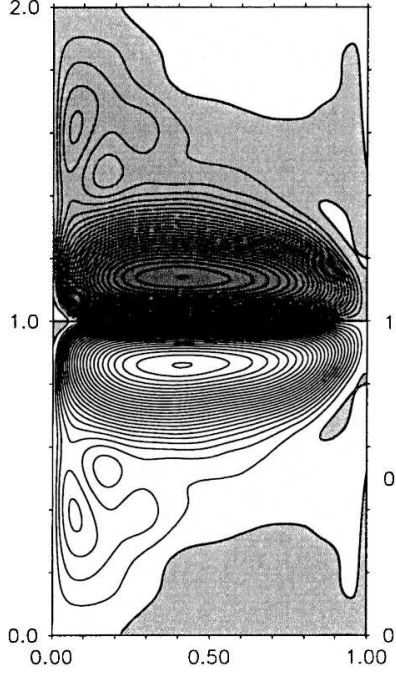

a.

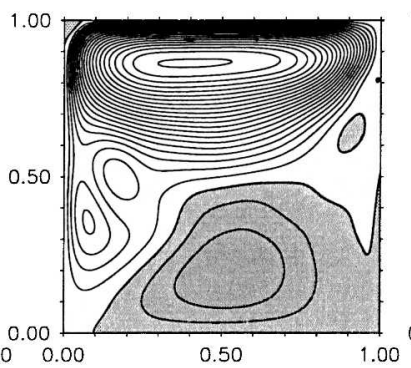

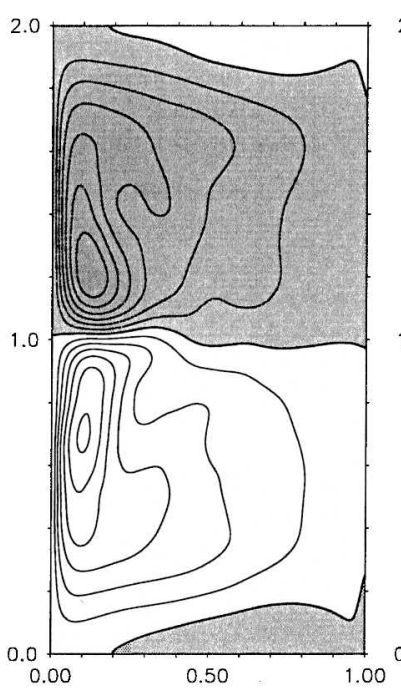

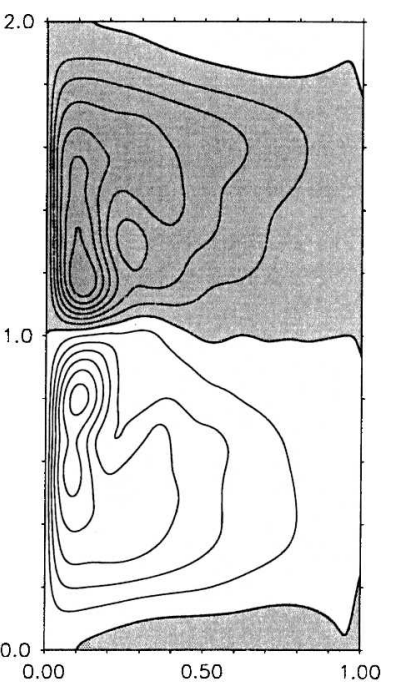

FIG. 10. Comparison of the time-mean streamfunction for no-slip $\mathrm{Re}=5$ calculations. (a), (c) Averages over different time intervals for a calculation with an at-rest (symmetric) initial condition. (a) The time mean over an interval just before exact antisymmetry is broken by numerical errors $(t=2000$ to 2750), and (c) averaged over the time interval $t=8990$ to 13710 . (b) The time-mean streamfunction for the single-gyre calculation. (d) The time-mean streamfunction for the calculation with symmetry-breaking initial conditions. The contour interval is 0.2 and shading indicates negative streamfunction.

has occurred (as in Fig. 10c); $\mathrm{Nu}_{\zeta}$ is $10-15$ times as large after equilibration than just before symmetry breaks. ${ }^{7}$

Thus, qualitatively different multiple equilibria exist in the time-dependent double gyre, and the availability of each equilibrium depends on the eddies and their symmetry. The difference between the single gyre and the double gyre with no-slip conditions on solid boundaries is not due to an intergyre vorticity flux. Rather it is the lack of sinuous modes in the single gyre that prohibits equilibria available to the double-gyre model. Figure 12 is an attempt to map the phase space of these equilibria. It shows the trajectory of the different spinups as a function of maximum and minimum timesmoothed streamfunction. Note how the initial symmetric double gyre first approaches a state with maximum and minimum streamfunction equal and opposite (gyre antisymmetry) at nearly the same magnitude as the single-gyre maximum streamfunction. Then the symmetry breaks and its trajectory moves off of the diagonal and into the same region as the initial asymmetric double gyre.

The removal of sinuous modes by using resting initial conditions provides compelling evidence as to the difference between the single-gyre and double-gyre models in no-slip calculations (i.e., when there is no inter-

\footnotetext{
${ }^{7}$ The duration of the single-gyre regime before symmetry breaks is too short for a better estimate.
}

gyre eddy flux). The specific case of resting initial conditions is only of limited direct applicability (e.g., decreasing the time to equilibration of double-gyre calculations), but reveals an important fact about eddy fluxes: vorticity flux efficiency plays as large a role as vorticity sinks and sources in determining circulation strength. In a more complicated model than the one here, such as a general circulation ocean model, the circulation strength will similarly depend on what type and how well resolved the eddies present are, even if the vorticity sources and sinks are correct.

\section{d. Adding bottom friction}

One might suppose that with increased bottom friction, the difference between slip and no-slip multiplegyre calculations would diminish as lateral frictionand hence boundary conditions required by lateral friction-become less important. However, calculations including bottom friction reveal that the frictional boundary conditions always affect the intergyre vorticity flux (Figs. 13a-f and Table 1). Even when Re is substantially increased (lowering lateral viscosity) and $\delta_{S}$ increased, there is still a large difference between slip and no-slip eddy fluxes (Figs. 13e-f and Table 1). Ultimately, no matter how small the lateral friction coefficient is, the boundary current continues to transport both positive and negative relative vorticity in no-slip calculations, so the net transport is small (per Stewart 1964, 1989). 


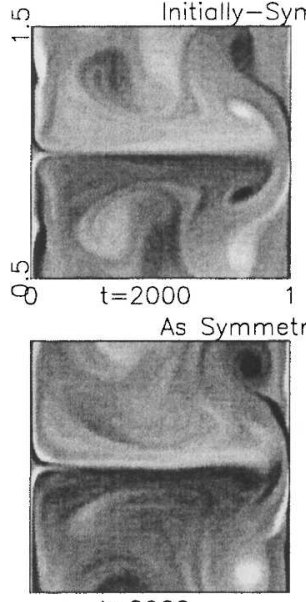

$t=2900$

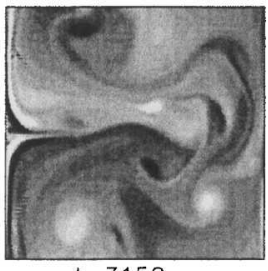

$t=3150$

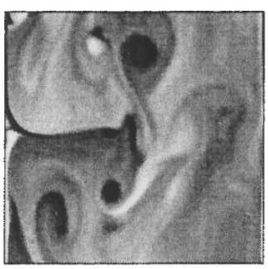

$t=3400$

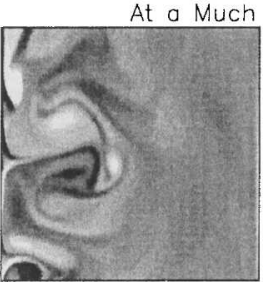

$t=9100$

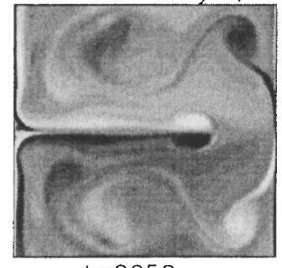

$t=2050$

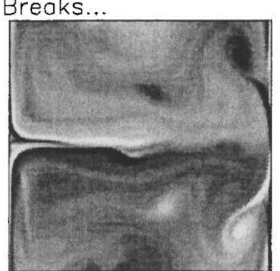

$t=2950$

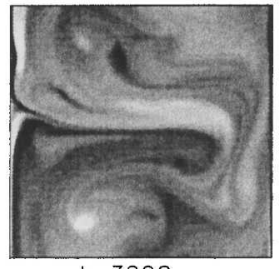

$t=3200$

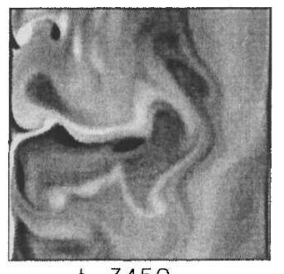

$t=3450$

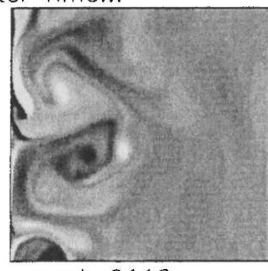

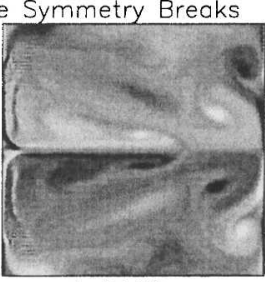

$t=2100$

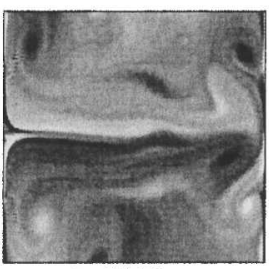

$t=3000$

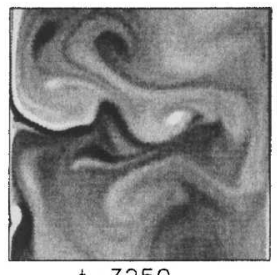

$t=3250$

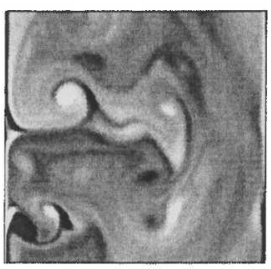

$t=3500$

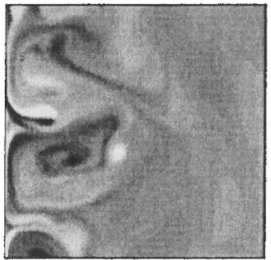

$t=9120$

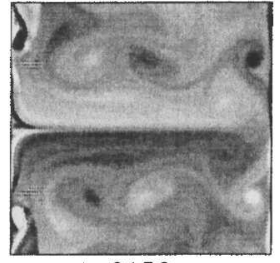

$t=2150$

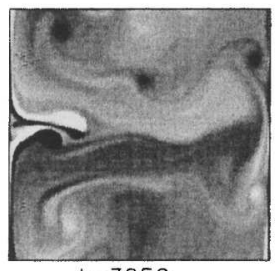

$t=3050$

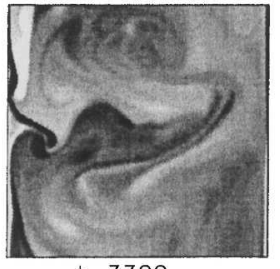

$t=3300$

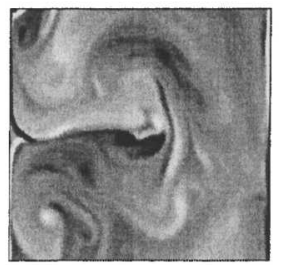

$t=3550$

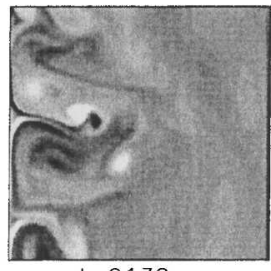

$t=9130$

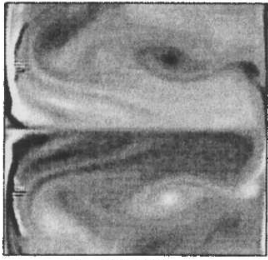

$t=2200$

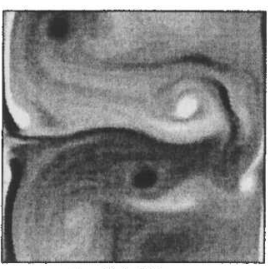

$t=3100$

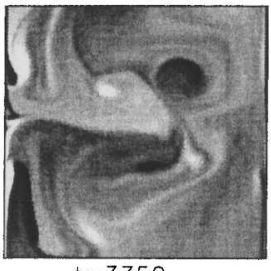

$t=3350$

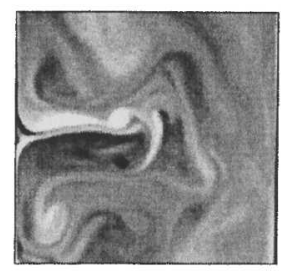

$t=3600$

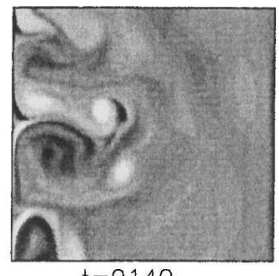

FIG. 11. Snapshots of relative vorticity in the region surrounding the jet $(0<x<1,0.5<y<1.5)$ of the double-gyre calculation begun with symmetric initial conditions before antisymmetry is broken (upper row), as antisymmetry is broken (second, third, and fourth rows), and snapshots after the circulation strength has reached equilibrium (bottom row) (lighter: more positive vorticity, darker: more negative).

Bottom friction does have an effect, however. The bottom friction fluxes vorticity across mean streamlines and out of the basin, as anticipated in (5). The addition of a small amount of bottom friction $\left(\delta_{S}=0.001\right)$ takes a share $(6 \%$; Table 1$)$ of vorticity flux and removal and thus reduces the burden for the intergyre eddy flux and lateral friction in the slip case (Fig. 2d versus Fig. 13a and Table 1).

The bottom friction also affects the instability processes that form eddies. In particular, the instabilities are damped, so the eddies grow more slowly and mix less efficiently than without bottom friction. This reduction in eddy efficiency is partly responsible for the reduction in intergyre vorticity flux in the slip case (Fig. $2 \mathrm{~d}$ versus Fig. 13a) and in the no-slip case (Fig. 13b versus Fig. 13c). Curiously, there are occasions when the reduction in eddy efficiency outweighs the increased ease of removal of vorticity by friction; the mean circulation may strengthen with increased drag! (cf. Fig. 13a with Fig. 13c.) Only if the eddy efficiency is 


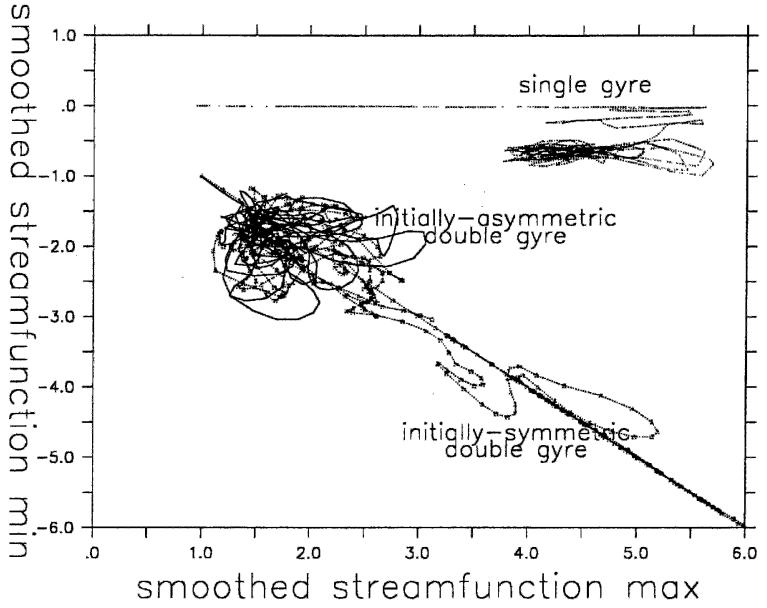

FIG. 12. Phase diagram of maximum and minimum streamfunction trajectory (smoothed with Hanning filter over 250 time unit window) during spinup of the single gyre, initially resting double gyre, and initially asymmetric double gyre shown in Fig. 10. The smoothing prohibits plotting the first few time intervals that connects the trajectories to the origin.

reduced does this result make sense, and a reduction in efficiency is observed (Fig. 13a versus Fig. 13c in Table 1).

The length of the gyre-dividing jet is strongly affected by the eddy efficiency as well (cf. $\mathrm{Nu}_{\zeta}$ in Table 1 with jet extents in Fig. 6 and Fig. 13). Holland and Schmitz (1985) and Marshall and Marshall (1992) argue that an eddy process determines the zonal penetration of the jet, and here it is clear that the friction affects the eddies that in turn affect the jet penetration. Le Provost and Verron (1987) present a scaling analysis of the penetration depth under varying bottom and lateral friction.

In conclusion, while bottom friction does affect the flow, especially the eddy growth rate and efficiency, the slip and no-slip calculations remain quite different in intergyre eddy flux, and the framework of eddy efficiency still aptly diagnoses the effects of eddies on the mean circulation.

\section{e. Ultimate vorticity removal}

Despite the increased efficiency of vorticity removal by the sinuous modes, at sufficiently high Reynolds number they are no longer capable of controlling the circulation strength (Fig. 14a). At this point, if the circulation is to be controlled then new physics must be introduced to the model, such as new vorticity sinks or increased eddy efficiency. For example, 1) vertical structure may further increase the efficiency of the eddy mixing as by baroclinic instability, or 2) sloping bottom topography may act as sink of vorticity in concert with bottom velocities (Hughes and de Cuevas 2001).

Increased viscosity in a narrow region near the boundary can be used as a proxy for these effects. FoxKemper and Pedlosky (2004) demonstrate the singlegyre circulation by boundary-enhanced viscosity. Here the same form of boundary-enhanced viscosity is used, but in a two-gyre calculation:

$\delta_{M}^{3}=\frac{\delta_{I}^{3}}{\operatorname{Re}_{i}}+\left(\frac{\delta_{I}^{3}}{\operatorname{Re}_{b}}-\frac{\delta_{I}^{3}}{\operatorname{Re}_{i}}\right) \times\left[e^{-x / \delta_{d}}+e^{-(1-x) / \delta_{d}}\right]$,

and

$\delta_{d} \equiv \frac{\delta_{I}}{\sqrt{\mathrm{Re}_{i}}}$.

In (11), $\delta_{M}$ varies so that the Reynolds number smoothly changes from a constant boundary Reynolds number $\left(\mathrm{Re}_{b}\right)$ to a constant interior Reynolds number
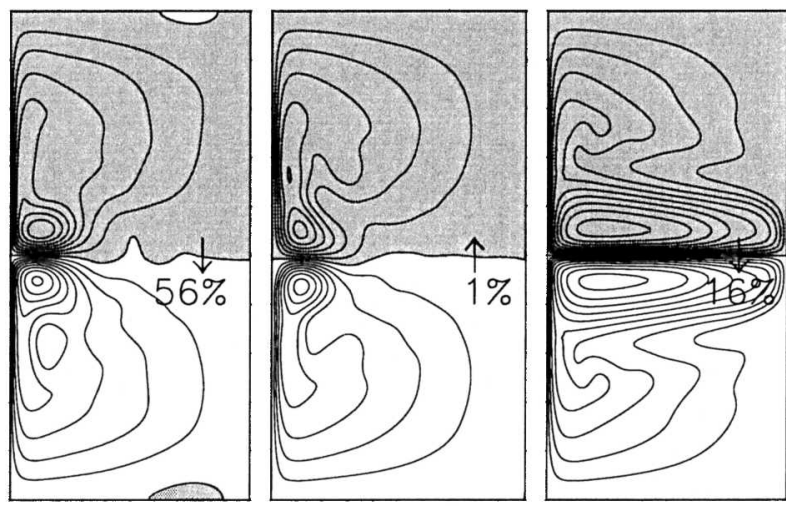

Re=5, $d_{s}=0.001$, Slip b. $\operatorname{Re}=5, d_{s}=0.001$, No-Slip c. $\operatorname{Re}=5, d_{s}=0.003$. Slip
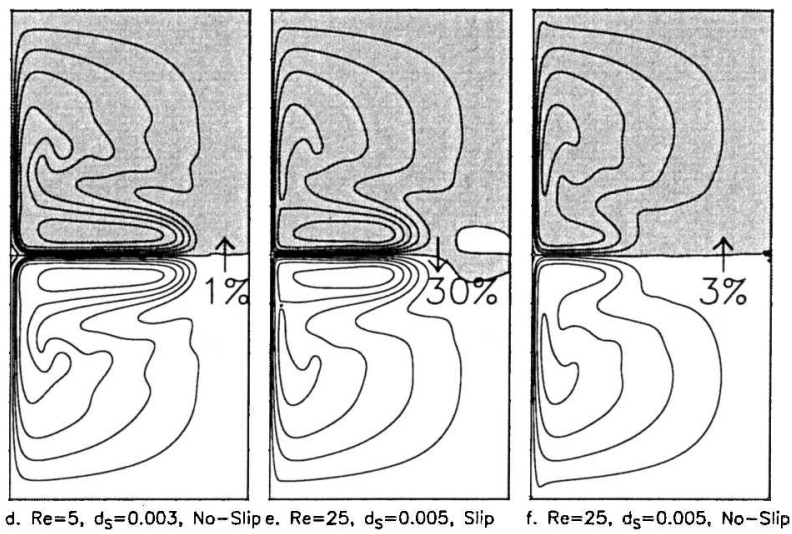

FIG. 13. Comparison of the time-mean streamfunction and intergyre eddy flux for calculations with bottom friction. The contour interval is 0.2 , and shading indicates negative streamfunction. 


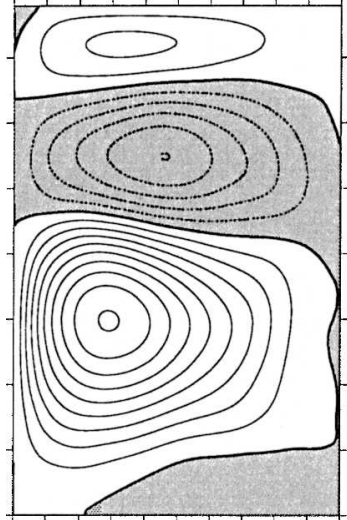

о. $\operatorname{Re}_{\mathrm{i}}=\operatorname{Re}_{\mathrm{b}}=25$

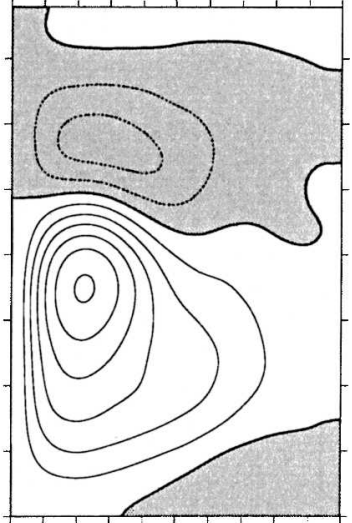

b. $R e_{i}=25, R e_{b}=0.25$
FIG. 14. Time-mean streamlines of the (a) no-slip, two-gyre calculation with constant viscosity $\left(\mathrm{Re}=25, \delta_{I}=0.06\right)$ and $(\mathrm{b})$ no-slip, two-gyre calculation with boundary-enhanced viscosity $\left(\operatorname{Re}_{b}=0.25, \operatorname{Re}_{i}=25, \delta_{I}=0.06\right)$. The contour interval is 0.2 , and shading indicates negative streamfunction.

$\left(\mathrm{Re}_{i}\right)$. Figure 14b shows that a narrow region ${ }^{8}$ of increased viscosity in the two-gyre calculation controls the two-gyre circulation as well. The increased viscosity is associated with increased eddy efficiency for the streamlines away from the boundary (Fig. 9).

\section{Summary and discussion}

The wind-driven barotropic ocean model is quite simple, but it is useful as a toy duplicating the real ocean's dynamics. Multiple gyres have been studied for almost the whole history of such models (Munk and Carrier 1950), but the importance of multiple gyres on eddy dynamics proposed by Harrison and Holland (1981) and Marshall (1984) continues to provide interesting results.

\section{a. Intergyre fluxes and boundary current overshoot}

The boundary conditions play an unexpectedly important role, as both dissipative meandering and permanent fluid exchange are initiated by a boundary current overshoot. Of course, neither slip nor no-slip boundary conditions correctly represent the complicated multiple-scale interaction of the real ocean and its boundary. However, the ocean's western boundary currents have their maximum velocity in the center (as in no-slip calculations), rather than on one side (as in slip calculations), and the separation point migrates little along the coast. Thus, the intergyre eddy flux possibilities seem limited.

\footnotetext{
${ }^{8}$ The increased viscosity scale is narrower by a factor of 5 than the inertial boundary layer scale here.
}

The correspondence between alternating overshooting boundary currents in the slip, double-gyre model and a permanent overshooting boundary current in the slip, two-gyre model nicely relates the global vorticity balance to the local confrontation of two opposing boundary currents. Categorizing these effects differently is in part an error of diagnosis, as the alternating boundary current is only categorized as an "eddy" effect due to the exact antisymmetry of the double-gyre model. In fact, the physics of the overshooting boundary current is identical in the two cases.

As to the difference between the boundary current overshoot and intergyre vorticity flux in no-slip and slip calculations, two key results offer explanation. First, the cancellatory relative vorticity in the no-slip boundary current limits the intergyre vorticity flux (Fig. 5 and Stewart 1964, 1989). Second, the tendency for the stronger of two colliding boundary currents to over/undershoot with slip/no-slip boundary conditions (Cessi 1991) provides the additional distinction between the models.

\section{b. The important, revised role of eddy vorticity fluxes in the barotropic gyre}

Because of the differences in the boundary current from which they form, no-slip and slip eddies have quite different vorticity fluxes, and thus no-slip and slip calculations strike quite different equilibrium vorticity budgets. The eddies in the multiple-gyre, no-slip calculations play the same role as those in the single-gyre calculations here and in Fox-Kemper and Pedlosky (2004). That is, they allow a flux of vorticity across mean streamlines. Thus, the wind input can be removed from the basin without requiring every streamline pass through a frictional region or requiring an unrealistically large circulation. In slip calculations, the eddies flux vorticity primarily to the other gyre, both by dissipative meandering and fluid exchange. However, this flux is restricted to calculations with slippery boundary conditions. Often, general circulation models are written to allow for only one type of frictional boundary condition or run with insufficient resolution to resolve the positive and negative relative vorticity in the no-slip boundary current, the evidence of the disparate behavior of this simple model under different boundary conditions accentuates how perilous these practices may be.

\section{c. The eddy transport efficiency}

It is not only the removal of vorticity that is important in controlling the circulation, but the efficiency of the eddies in transporting vorticity to the removal region. Less efficient eddies require larger vorticity gradients from which to form and across which to mix. Seemingly paradoxical results are explained by changes 
in eddy efficiency, such as the calculations (Fig. 13a versus Fig. 13c) where increasing the drag strengthened the mean flow.

In the no-slip calculations, it is the inefficiency of the single-gyre eddies that allows the circulation to be so much larger than in the multiple-gyre calculations. The sinuous modes are significantly more efficient than the varicose modes, so the resulting mean circulation is much weaker when they are present.

Observations of "meanders" are common in the Gulf Stream Extension and Kuroshio Extension (e.g., Hogg 1994; Spall and Robinson 1990; Cornillon et al. 1994). The sinuous modes are the analog of these meanders in this simple model. While it is difficult to imagine a real ocean where sinuous modes were prohibited as in the spinup from rest of the double gyre, the efficiency of the sinuous modes makes them rather important to observe and analyze as they seem to be the most likely eddies to play a role in fluxing near- conservative properties such as vorticity.

Other aspects of the three-dimensional problem will require adaptation of the methods used here. The budget of vorticity becomes a budget of vorticity for each isopycnal layer (Haynes and McIntyre 1987). Momentum fluxes can be achieved by layer-to-layer "form drag" (Rhines and Holland 1979), and this will have a corresponding vorticity flux within each layer. Furthermore, the pattern of streamlines may be much more complicated in three dimensions (Yang and Liu 1997). Obviously, the cross-jet transport of vorticity is quite different in this case, especially when the isopycnals outcrop into the mixed layer along the jet. Progress on the three-dimensional problem (Holland and Rhines 1980; Lozier and Riser 1990; Bower and Lozier 1994; Berloff 2005; Yuan et al. 2004) has been substantial, but the problem of determining the circulation at each level is not fully addressed, in part because of the unexpected importance of boundary conditions and the role of eddy flux efficiency addressed here.

There has been a tacit assumption in eddy flux analysis: to increase eddy fluxes one must strengthen the circulation or alter the sources and sinks of vorticity. This is not true when multiple types of eddies of differing efficiency are available. In that case, the eddy fluxes can be increased by increasing the eddy efficiency. The lack of topography and vertical structure in this model leaves out many important effects and potentially efficient eddy processes that may be studied via a similar approach in the future. It is clear from the evidence presented here that including slightly different eddiesfrom replacing a parameterization with resolved eddies or from introducing new physics such as baroclinic instability or nonhydrostatic effects-may have a pro- found effect on even large-scale flow and the global vorticity budget.

Acknowledgments. The author thanks J. Pedlosky and P. Rizzoli for their important comments and suggestions on this work. Thanks also are given to S. Jayne who contributed almost all of the computer resources. Very useful comments were provided by B. Arbic, P. Berloff, J. Marshall, J. Franklin, G. Flierl, J. Russell, M. Spall, and V. Sheremet. During the course of this research and manuscript preparation, the author was supported by an ONR-supported NDSEG fellowship, an MIT Presidential Fellowship, NSF OCE 9910654, a Princeton University/GFDL fellowship, and a NOAA Climate and Global Change postdoctoral fellowship managed by UCAR.

\section{REFERENCES}

Balmforth, N. J., and C. Piccolo, 2001: The onset of meandering in a barotropic jet. J. Fluid Mech., 449, 85-114.

Becker, J. M., and R. Salmon, 1997: Eddy formation on a continental slope. J. Mar. Res., 55, 181-200.

Berloff, P. S., 1998: On the stability of the wind-driven circulation. J. Mar. Res., 59, 937-993.

— 2005: On dynamically consistent eddy fluxes. Dyn. Atmos. Oceans, in press.

—, and S. P. Meacham, 1998: The dynamics of a simple baroclinic model of the wind-driven circulation. J. Phys. Oceanogr., 28, 361-388.

_ J. C. McWilliams, and A. Bracco, 2002: Material transport in oceanic gyres. Part I: Phenomenology. J. Phys. Oceanogr., 32, 764-796.

Bower, A. S., and M. S. Lozier, 1994: A closer look at particle exchange in the gulf stream. J. Phys. Oceanogr., 24, 13991418.

Carrier, G. F., and A. R. Robinson, 1962: On the theory of the wind-driven ocean circulation. J. Fluid Mech., 12, 49-80.

Cessi, P., 1991: Laminar separation of colliding western boundary currents. J. Mar. Res., 49, 697-717.

_, and G. R. Ierley, 1990: Symmetry-breaking multiple equilibria in quasi-geostrophic, wind-driven flows. J. Phys. Oceanogr., 25, 1196-1205.

Chang, K.-I., M. Ghil, K. Ide, and C.-C. A. Lai, 2001: Transition to aperiodic variability in a wind-driven double-gyre circulation model. J. Phys. Oceanogr., 31, 1260-1286.

Charney, J. G., 1955: The Gulf Stream as an inertial boundary layer. Proc. Natl. Acad. Sci., 41, 731-740.

Cornillon, L., T. Lee, and G. Fall, 1994: On the probability that a Gulf Stream meander crest detaches to form a warm core ring. J. Phys. Oceanogr., 24, 159-171.

Coulliette, C., and S. Wiggins, 2001: Intergyre transport in a winddriven, quasigeostropic double gyre: An application of lobe dynamics. Nonlinear Proc. Geophys., 8, 69-94.

Dijkstra, H. A., and C. A. Katsman, 1997: Temporal variability of the wind-driven quasi-geostrophic double gyre ocean circulation: Basic bifurcation diagrams. Geophys. Astrophys. Fluid Dyn., 85, 195-232.

Edwards, C. A., and J. Pedlosky, 1998: Dynamics of nonlinear cross-equatorial flow. Part I: Potential vorticity transformation. J. Phys. Oceanogr., 28, 2382-2406. 
Fofonoff, N. P., 1954: Steady flow in a frictionless homogenous ocean. J. Mar. Res., 13, 254-262.

Fox-Kemper, B., 2003: Friction and eddies: Removal of vorticity from the wind-driven gyre. Ph.D. thesis, MIT/WHOI, 200306, $310 \mathrm{pp}$.

— 2004: Wind-driven barotropic gyre. II: Effects of eddies and low interior viscosity. J. Mar. Res., 62, 195-232.

—_, and J. Pedlosky, 2004: Wind-driven barotropic gyre. I: Circulation control by eddy vorticity fluxes to an enhanced removal region. J. Mar. Res., 62, 169-193.

— , R. Ferrari, and J. Pedlosky, 2003: On the indeterminacy of rotational and divergent eddy fluxes. J. Phys. Oceanogr., 33, 478-483.

Ghil, M., Y. Feliks, and L. U. Sushama, 2002: Baroclinic and barotropic aspects of wind-driven ocean circulation. Physica D, 167, 1-35.

Griffiths, R.W., 1998: Linear theory of the effect of a sloping boundary on circulation in a homogeneous laboratory model. J. Mar. Res., 56, 75-86.

—_, and G. Veronis, 1997: A laboratory study of effects of a sloping side boundary on wind-driven circulation in a homogeneous ocean model. J. Mar. Res., 55, 1103-1126.

Haidvogel, D. B., J. C. McWilliams, and P. R. Gent, 1992: Boundary current separation in a quasigeostrophic, eddy-resolving ocean circulation model. J. Phys. Oceanogr., 22, 882-902.

Harrison, D. E., and W. R. Holland, 1981: Regional eddy vorticity transport and the equilibrium vorticity budgets of a numerical model ocean circulation. J. Phys. Oceanogr., 11, 190-208.

Haynes, P., and M. E. McIntyre, 1987: On the evolution of vorticity and potential vorticity in the presence of diabatic heating and frictional or other forces. J. Atmos. Sci., 44, 828-841.

Hogg, N. G., 1994: Observations of gulf stream meander-induced disturbances. J. Phys. Oceanogr., 24, 2534-2546.

Holland, W. R., and P. B. Rhines, 1980: An example of eddyinduced ocean circulation. J. Phys. Oceanogr., 10, 1010-1031.

_ midlatitude jets. J. Phys. Oceanogr., 15, 1859-1875.

Hughes, C. W., and B. A. de Cuevas, 2001: Why western boundary currents in realistic oceans are inviscid: A link between form stress and bottom pressure torques. J. Phys. Oceanogr., 31, 2871-2885.

Ierley, G. R., 1987: On the onset of recirculation in barotropic general circulation models. J. Phys. Oceanogr., 17, 2366-2374.

— a nonlinear boundary-layer problem. Stud. Appl. Math., 75, $1-36$.

- and V. A. Sheremet, 1995: Multiple solutions and advectiondominated flows in the wind-driven circulation. Part I: Slip. J. Mar. Res., 53, 703-737.

Il'in, A. M., and V. M. Kamenkovich, 1964: The structure of the boundary layer in a two-dimensional model of ocean currents (in Russian). Okeanologiya, 4, 756-769.

Le Provost, C., and J. Verron, 1987: Wind-driven ocean circulation transition to barotropic instability. Dyn. Atmos. Oceans, 11, 175-201.

Lozier, M. S., and S. C. Riser, 1990: Potential vorticity sources and sinks in a quasi-geostrophic ocean: Beyond western boundary currents. J. Phys. Oceanogr., 20, 1608-1627.

Marshall, D., and J. Marshall, 1992: Zonal penetration scale of midlatitude oceanic jets. J. Phys. Oceanogr., 22, 1018-1032.

Marshall, D. P., and J. C. Stephens, 1998: On the insensitivity of the wind-driven circulation to bottom topography. J. Mar. Res., 59, 1-27.
Marshall, J. C., 1984: Eddy mean flow interaction in a barotropic ocean model. Quart. J. Roy. Meteor. Soc., 100, 573-590.

Moro, B., 1987: On the inertial motion of a homogeneous ocean. Dyn. Atmos. Oceans, 11, 1-17.

_, 1988: On the nonlinear Munk model. Part I: Steady flows. Dyn. Atmos. Oceans, 12, 259-287.

, 1990: On the nonlinear Munk model. Part II: Stability. Dyn. Atmos. Oceans, 14, 203-227.

Munk, W. H., 1950: On the wind-driven ocean circulation. J. Meteor., 7, 79-93.

_, and G. F. Carrier, 1950: The wind-driven circulation in ocean basins of various shapes. Tellus, 2, 158-167.

Nauw, J. J., and H. A. Dijkstra, 2001: The origin of low-frequency variability of double-gyre wind-driven flows. J. Mar. Res., 59, 567-597.

Niiler, P. P., 1966: On the theory of the wind-driven ocean circulation. Deep-Sea Res., 13, 597-606.

Plumb, R. A., 1982: A new look at the energy cycle. J. Atmos. Sci., 40, 1669-1688.

Pratt, L. J., M. S. Lozier, and N. Beliakova, 1995: Parcel trajectories in quasigeostrophic jets: Neutral modes. J. Phys. Oceanogr., 25, 1451-1466.

Primeau, F., 1998: Multiple equilibria of a double-gyre ocean model with super-slip boundary conditions. J. Phys. Oceanogr., 28, 2130-2147.

Rhines, P. B., and W. R. Holland, 1979: A theoretical discussion of eddy-driven mean flows. Dyn. Atmos. Oceans, 3, 289-325.

Rogerson, A. M., P. D. Miller, L. J. Pratt, and C. K. R. T. Jones, 1999: Lagrangian motion and fluid exchange in a barotropic meandering jet. J. Phys. Oceanogr., 29, 2635-2655.

Scott, R. B., and D. N. Straub, 1998: Small viscosity behavior of a homogeneous, quasigeostrophic, ocean circulation model. $J$. Mar. Res., 56, 1225-1258.

Sheremet, V. A., G. R. Ierley, and V. M. Kamenkovich, 1997: Eigenanalysis of the two-dimensional wind-driven ocean circulation problem. J. Mar. Res., 55, 57-92.

Spall, M. A., and A. R. Robinson, 1990: Regional primitive equation studies of the gulf stream meander and ring formation region. J. Phys. Oceanogr., 20, 985-1016.

Speich, S., H. A. Dijkstra, and M. Ghil, 1995: Successive bifurcations in a shallow-water model applied to the wind-driven ocean circulation. Nonlinear Process. Geophys., 2, 241-268.

Stewart, R. W., 1964: The influence of friction on inertial models of oceanic circulation. Studies on Oceanography: A Collection of Papers Dedicated to Koji Hidaka, K. Yoshida, Ed., University of Washington Press, 3-9.

, 1989: The no-slip constraint and ocean models. Atmos.Oceans, 27, 542-552.

Stommel, H. M., 1948: The westward intensification of winddriven ocean currents. Trans., Amer. Geophys. Union, 29, 202-206.

Sverdrup, H. U., 1947: Wind-driven currents in a baroclinic ocean; with application to the equatorial currents of the eastern $\mathrm{Pa}$ cific. Proc. Natl. Acad. Sci., 33, 318-326.

van der Vaart, P. C. F., H. M. Schuttelaars, D. Calvete, and H. A. Dijkstra, 2002: Instability of time-dependent wind-driven ocean gyres. Phys. Fluids, 14, 3601-3615.

Yang, H., and Z. Liu, 1997: The three-dimensional chaotic transport and the great ocean barrier. J. Phys. Oceanogr., 27, 1258-1273.

Yuan, G.-C., L. J. Pratt, and C. K. R. T. Jones, 2004: Cross-jet transport and mixing in a $2 \frac{1}{2}$-layer model. J. Phys. Oceanogr., 34, 1991-2005. 